\title{
Intrauterine Device Migration
}

Anisha Turner, MD

\section{A 33-year-old woman with a 2-year history of rectal bleeding presented following a recent episode that was accompanied by weakness.}

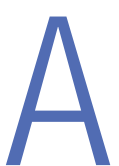

lthough intrauterine devices (IUDs) are a mainstay of reversible contraception, they do carry the risk of complications, including septic abortion, abscess formation, ectopic pregnancy, bleeding, and uterine perforation. ${ }^{1}$ Although perforation is a relatively rare complication, occurring in 0.3 to 2.6 per 1,000 insertions for levonorgestrelreleasing intrauterine systems and 0.3 to 2.2 per 1,000 insertions for copper IUDs, it can lead to serious complications, including IUD migration to various sites. ${ }^{2}$ Most patients with uterine perforation and IUD migration present with abdominal pain and bleeding; however, $30 \%$ of patients are

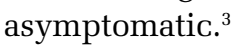

This article presents the case of a young woman who was diagnosed with IUD migration into the abdominal cavity. I discuss the management of this uncommon complication, and stress the importance of adequate education for both patients and health care providers regarding proper surveillance.

\section{Case}

A 33-year-old woman (gravida 4, para 4, live 4) presented to our ED for evaluation of rectal bleeding that she had experienced intermittently over the past 2 years. She reported that the first occurrence had been 2 years ago, starting a few weeks after she had a cesarean delivery. The patient described the initial episode as bright red blood mixed with stool. She stated that subsequent episodes had been intermittent, felt as if she were "passing rocks" through her abdomen and rectum, and were accompanied by streaks of blood covering her stool. The day before the patient presented to the ED, she had experienced a second episode of a large bowel movement mixed with blood and accompanied by weakness, which prompted her to seek treatment.

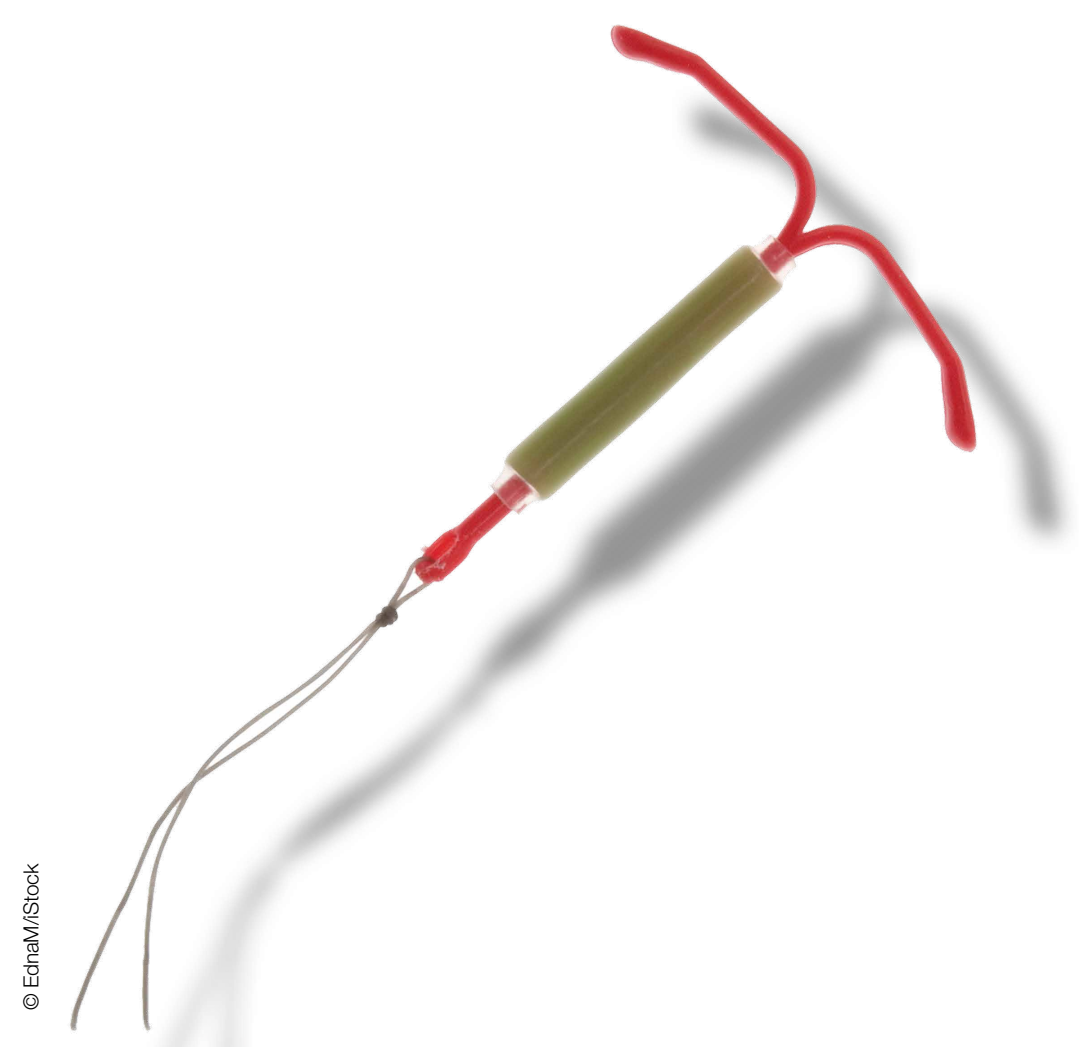

Dr Turner is a third-year family medicine and emergency medicine resident, Louisiana State University Health Sciences Center-Shreveport. 


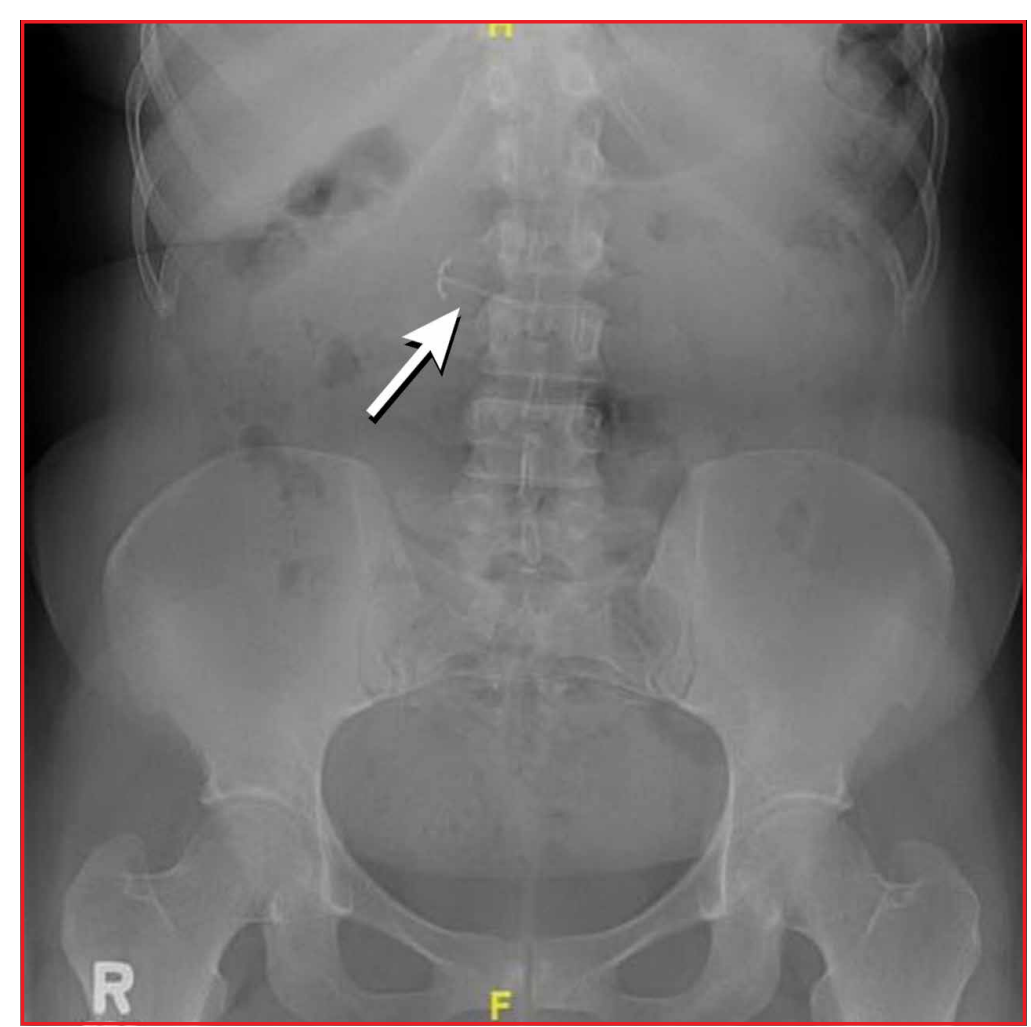

Figure 1. Abdominal X-ray imaging reveals a nonobstructive stool pattern and a foreign body, likely in the abdominal cavity, which appears to be an intrauterine device (white arrow). dominal examination exhibited normoactive bowel sounds with diffuse lower abdominal tenderness to deep palpation, but without rebound, guarding, or distension. Rectal examination revealed a small internal hemorrhoid at the 6 o'clock position (no active bleeding) and an external hemorrhoid with some tenderness to palpation; the external hemorrhoid was not thrombosed, had no signs of infection, and was the same color as the surrounding skin.

A fecal occult blood screen was negative, and a serum pregnancy test was also negative. Complete blood count, basic metabolic profile, and urinalysis were all unremarkable and within normal ranges. Abdominal X-ray revealed a nonobstructive stool pattern and a foreign body, likely in the abdominal cavity, which appeared to be an IUD (Figure 1). Computed tomography (CT) scans of the abdomen and pelvis without contrast were performed to accurately locate the foreign body and to assess for any complications. The CT scans revealed an IUD outside of the uterus, between loops of the transverse colon within the left midabdomen (Figure 2). There were no signs of infection, fluid, or free air. There were also findings of colonic diverticula and narrowed lumen, which were suggestive of diverticulosis.

The patient stated that the IUD had been placed several months after the vaginal birth of her third child. She continued to have normal menstrual periods with the IUD in place. Seven years later, she became pregnant with her fourth child, who was delivered via cesarean, secondary to fetal malpositioning. The IUD was not removed during the cesarean delivery.

Based on the CT scan findings, gynecology services was consulted, and the gynecologist recommended immediate followup in a gynecology clinic. The patient was discharged on a bowel regimen. She was assessed in a gynecology clinic 4 days later, where she was found to have a mobile retroverted uterus without tenderness or signs of infection. She underwent explor- 
atory laparoscopy, during which the IUD was removed from the omentum in the left upper abdomen without complications.

\section{Discussion}

The IUD has had great acceptance among women since the 1960s. According to the World Health Organization, approximately $14.3 \%$ of women used an IUD in $2009 .{ }^{4}$ Although complications are rare, the most serious are perforation of the uterus and migration of the IUD into adjacent organs. ${ }^{1}$

Risk factors of uterine perforation include clinician inexperience in IUD placement, an immobile uterus, a retroverted uterus, and the presence of a myometrial defect. ${ }^{4}$ Heinemann et $\mathrm{al}^{2}$ also suggested that breastfeeding and IUD placement soon after a delivery ( $\leq 36$ weeks) are independent risk factors, and the presence of both factors has an additive increase in risk of perforation.

Primary rupture of the uterus has been reported at the time of IUD insertion, but secondary or delayed rupture is more common and seems to be due to the spasms of the uterus. ${ }^{5}$ Although $85 \%$ of perforations do not affect other organs, the remaining $15 \%$ lead to complications in the adjacent visceral organs. ${ }^{6}$ The most frequent sites of migration are to the omentum $(26.7 \%)$, pouch of Douglas $(21.5 \%)$, large bowel $(10.4 \%)$, myometrium $(7.4 \%)$, broad ligament (6.7\%), abdominal cavity (5.2\%), adhesion to ileal loop serosa $(4.4 \%)$ or large bowel serosa (3.7\%), and mesentery ( $3 \%)^{7}$ Rare sites are to the appendix, abdominal wall, ovary, and bladder. ${ }^{7}$

Intrauterine device migration should be suspected in patients who become pregnant after IUD placement (as was the case for our patient), when the "threads" or string cannot be located while attempting to remove an IUD, or when a patient has an "expulsed" IUD without observation of the device thereafter. Even though expulsion of the device happens in approximately 8 per 1,000 insertions, uterine perforation is also a possibility in the case of a "lost" IUD. ${ }^{8}$

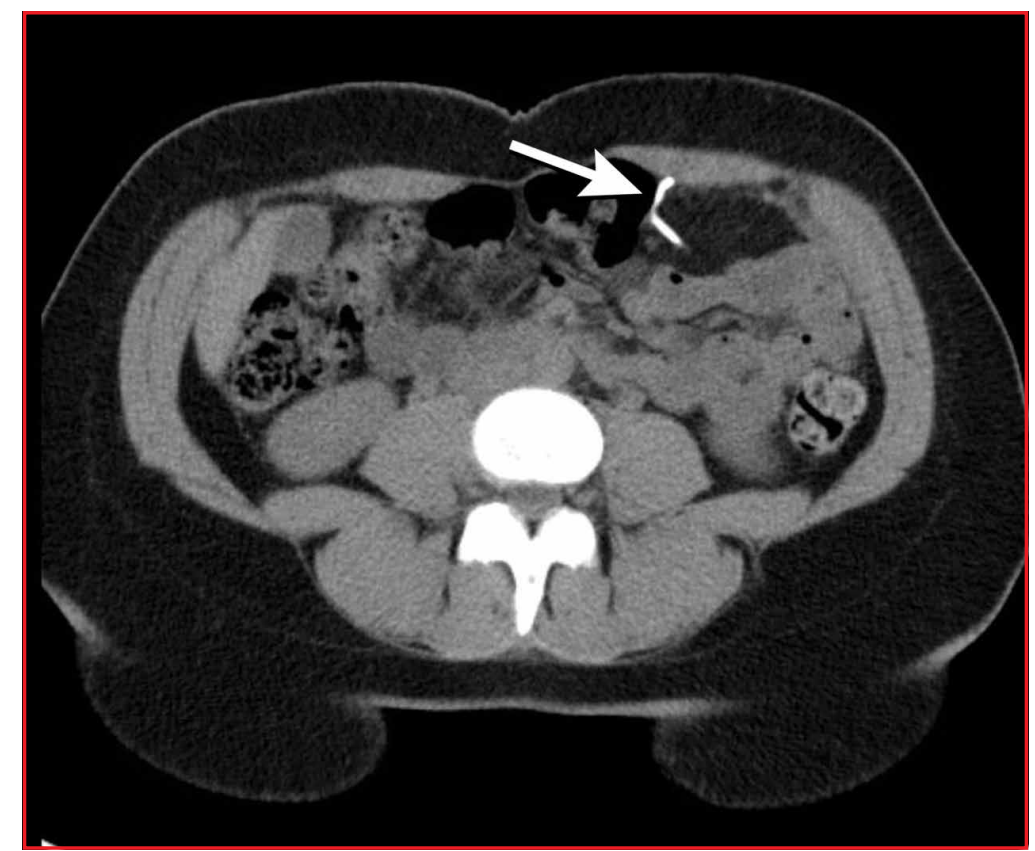

Figure 2. Computed tomography scan reveals an intrauterine device outside of the uterus, between loops of the transverse colon within the left midabdomen (white arrow).

When a lost IUD is suspected, a pelvic examination should be performed to assess for threads or string location. If unsuccessful, ultrasound or plain abdominal radiographic imaging may be used to locate the IUD. Once IUD migration has been confirmed, cross-sectional imaging such as CT scans or magnetic resonance imaging (MRI) is suggested to rule out adjacent organ involvement before considering surgical removal. ${ }^{4}$ If colonic involvement is suspected, colonoscopy can be used to confirm the diagnosis before operative removal. ${ }^{4}$

Although management of a migrated IUD in an asymptomatic patient is controversial, there appears to be a consensus that all extrauterine devices should be removed unless the patient's surgical risk is excessive. ${ }^{1,5,9}$ Retrieval of an IUD can be performed by laparotomy or laparoscopy. ${ }^{10,11}$

To avoid these complications and interventions, IUDs should be inserted by an appropriately trained professional, after proper patient selection. These devices should be monitored by periodic examina- 
tions, either by medical professionals or by well-informed patients. This can be done by either checking for the threads or string in the cervical opening or by ultrasound imaging to confirm the location of the IUD.

\section{Conclusion}

Although many patients with uterine perforation and IUD migration present with symptoms, approximately $30 \%$ are asymptomatic. ${ }^{3}$ If a patient has a lost IUD and the threads or string is not visible during pelvic examination, appropriate work-up, including transvaginal or transabdominal ultrasound or radiographs, should be obtained to confirm the position of the IUD. If IUD migration is suspected, cross-sectional imaging, such as CT scans or MRI, is recommended to rule out adjacent organ involvement before considering surgical removal. ${ }^{4}$

Even though only $15 \%$ of migrated IUDs lead to complications in the adjacent visceral organs, ${ }^{6}$ surgical removal of the IUD is advised regardless of the presence of symptoms or identified complications. Importantly, to prevent the delayed diagnosis and morbidity of IUD migration, patients with IUDs should be educated about the possibility of migration and the importance of regular self-examination for missing threads or string.

\section{References}

1. Hoşcan MB, Koşar A, Gümüştaş U, Güney M. Intravesical migration of intrauterine device resulting in pregnancy. Int J Urol. 2006;13(3):301-302.

2. Heinemann K, Reed S, Moehner S, Minh TD. Risk of uterine perforation with levonorgestrel-releasing and copper intrauterine devices in the European Active Surveillance Study on Intrauterine Devices. Contraception. 2015;91(4):274-279.

3. Singh SP, Mangla D, Chawan J, Haq AU. Asymptomatic presentation of silent uterine perforation by $\mathrm{Cu}-\mathrm{T}$ 380A: a case report with review of literature. Int J Reprod Contracept Obstet Gynecol. 2014;3(4):1157-1159.

4. Akpinar F, Ozgur EN, Yilmaz S, Ustaoglu O. Sigmoid colon migration of an intrauterine device. Case Rep Obstet Gynecol. 2014;2014:207659.

5. Rahnemai-Azar AA, Apfel T, Naghshizadian R, Cosgrove JM, Farkas DT. Laparoscopic removal of migrated intrauterine device embedded in intestine. JSLS. 2014;18(3).

6. Zakin D, Stern WZ, Rosenblatt R. Complete and partial uterine perforation and embedding following insertion of intrauterine devices. II. Diagnostic methods, prevention, and management. Obstet Gynecol Surv. 1981;36(8):401-417.

7. Gill RS, Mok D, Hudson M, Shi X, Birch DW, Karmali S. Laparoscopic removal of an intra-abdominal intrauterine device: case and systematic review. Contraception. 2012;85(1):15-18.

8. Paterson H, Ashton J, Harrison-Woolrych M. A nationwide cohort study of the use of the levonorgestrel intrauterine device in New Zealand adolescents. Contraception. 2009;79(6):433-438.

9. Gorsline JC, Osborne NG. Management of the missing intrauterine contraceptive device: report of a case. Am J Obstet Gynecol. 1985;153(2):228-229.

10. Mederos R, Humaran L, Minervini D. Surgical removal of an intrauterine device perforating the sigmoid colon: a case report. Int J Surg. 2008;6(6):e60-e62.

11. Chi E, Rosenfeld D, Sokol TP. Laparoscopic removal of an intrauterine device perforating the sigmoid colon: a case report and review of the literature. Am Surg. 2005;71(12):1055-1057. 